\title{
Correction: Effective in silico prediction of new oxazolidinone antibiotics: force field simulations of the antibiotic-ribosome complex supervised by experiment and electronic structure methods
}

Jörg Grunenberg ${ }^{* 1}$ and Giuseppe Licari ${ }^{*} 1,2$

\section{Correction}

\section{Address:}

${ }^{1}$ Institut für Organische Chemie, Hagenring 30, TU-Braunschweig, 38106 Braunschweig, Germany and ${ }^{2}$ Physical Chemistry Department, Sciences II, University of Geneva, 30, Quai Ernest Ansermet,

$\mathrm{CH}-1211$ Geneva 4, Switzerland

Email:

Jörg Grunenberg * - Joerg.Grunenberg@tu-bs.de; Giuseppe Licari ${ }^{*}$ -

Giuseppe.Licari@unige.ch

* Corresponding author

Keywords:

compliance constants; computational chemistry; drug design;

molecular recognition; relaxed force constants
Beilstein J. Org. Chem. 2016, 12, 608-610.

doi:10.3762/bjoc.12.59

Received: 21 March 2016

Accepted: 22 March 2016

Published: 31 March 2016

Associate Editor: P. R. Schreiner

(c) 2016 Grunenberg and Licari; licensee Beilstein-Institut. License and terms: see end of document.

This correction refers to Beilstein J. Org. Chem. 2016, 12, 415-428. doi:10.3762/bjoc.12.45

Our original publication contains an erratic number of predicted antibiotic structures in Scheme 2. With this Erratum we provide the corrected Scheme 2. 
<smiles>CC(C)NC[C@H]1CN(c2ccc(N3CCOCC3)c(Cl)c2)C(=O)O1</smiles>

Guest 8<smiles>O=C(N[C@@H]1CC12CN(c1ccc(N3CCOCC3)c(F)c1)C(=O)O2)c1ccccc1</smiles>

Guest 11<smiles>CC(C)N[C@H]1NC(=O)C[C@@]12CN(c1ccc(N3CCOCC3)c(F)c1)C(=O)O2</smiles><smiles>CC(=O)NC[C@H]1CN(c2cc(F)c(N3CCOCC3)c3ncccc23)C(=O)O1</smiles>

Guest 17<smiles>CC(C)N[C@H]1CCc2c1oc(=O)n2-c1ccc(N2CCOCC2)c(F)c1</smiles>

Guest 20<smiles>O=C1CC[C@H]([C@@H]2CN(c3ccc(N4CCOCC4)c(F)c3)C(=O)O2)N1</smiles>

Guest 23<smiles>CC(=O)Nc1cc[nH]c1[C@H]1CN(c2ccc(N3CCOCC3)c(F)c2)C(=O)O1</smiles>

Guest 26<smiles>CC(C)NC[C@H]1CN(c2ccc(N3CCOCC3)c(Br)c2)C(=O)O1</smiles>

Guest 9<smiles></smiles>

Guest 12<smiles>N[C@@H]1NC(=O)CO[C@]12CN(c1ccc(N3CCOCC3)c(F)c1)C(=O)O2</smiles>

Guest 15<smiles>O=C1O[C@@H](CNC2CC2)CN1c1cc(F)c(N2CCOCC2)c2ccccc12</smiles>

Guest 18<smiles>O=C1O[C@H]2[C@H](CC[C@H]2NC=C2CC2)N1c1ccc(N2CCOCC2)c(F)c1</smiles>

Guest 21<smiles>O=C1C=C[C@H]([C@@H]2CN(c3ccc(N4CCOCC4)c(F)c3)C(=O)O2)N1</smiles>

Guest 24<smiles>CC(C)NC(NC(=O)O)[C@H]1CN(c2ccc(N3CCOCC3)c(F)c2)C(=O)O1</smiles>

Guest 27<smiles>CCOCCNC[C@H]1CN(c2ccc(N3CCOCC3)c(F)c2)C(=O)O1</smiles><smiles>O=C1O[C@@H](CNC2CC2)CN1c1ccc(N2CCOCC2)c(I)c1</smiles>

Guest 10<smiles>CC(C)=C1NC(=O)C[C@@]12CN(c1ccc(N3CCOCC3)c(F)c1)C(=O)O2</smiles><smiles>N[C@@H]1NC(=O)CO[C@]12CN(c1ccc(N3CCOCC3)c(F)c1)C(=O)O2</smiles>

Guest 16<smiles>CC(C)NC[C@H]1CN(c2ccc(N3CCOCC3)c(F)c2F)C(=O)O1</smiles>

Guest 19<smiles>CC(C)NCC[C@@H]1CN(c2ccc(N3CCOCC3)c(F)c2)C(=O)O1</smiles>

Guest 22<smiles>O=C1O[C@@H](c2ccc[nH]2)CN1c1ccc(N2CCOCC2)c(F)c1</smiles>

Guest 25<smiles>CC(C)C=CNCC1(CNCC(C)C)CN(c2ccc(N3CCOCC3)c(F)c2)C(=O)O1</smiles>

Guest 28 


\section{License and Terms}

This is an Open Access article under the terms of the Creative Commons Attribution License

(http://creativecommons.org/licenses/by/2.0), which permits unrestricted use, distribution, and reproduction in any medium, provided the original work is properly cited.

The license is subject to the Beilstein Journal of Organic Chemistry terms and conditions:

(http://www.beilstein-journals.org/bjoc)

The definitive version of this article is the electronic one which can be found at:

$\underline{\text { doi: } 10.3762 / \text { bjoc. } 12.59}$ 